Gesnerus 65 (2008) 86-107

Essay Review

\title{
Körper- und Medizingeschichte des alten und mittelalterlichen Chinas, 1997-2007
}

\author{
Rudolf Pfister
}

Keywords: historiography of the body and medicine; ancient and medieval China; sexual body techniques; kallisthenics; Dunhuang medical manuscripts; innovation in Chinese medicine; medicine for women; paediatrics; ophthalmology

Die Körper- und Medizingeschichtsschreibung über das alte und mittelalterliche China hat sich differenziert:Von der Hinwendung zu «Klassikern» zu der Bearbeitung von Manuskriptquellen und Einzelschriften, von einer Tendenz der Umschreibung und Vereinheitlichung für die moderne Praxis $\mathrm{zu}$ einer Historisierung (etwa der Innovation) und der Periodisierung, von einem Dornröschenschlaf der Philologie zu einem zögerlich vollzogenen diesbezüglichen Aufwachen und einigen wenigen Übersetzungen mit medizingeschichtlichem Anspruch ${ }^{1}$. Die folgende kurze Darstellung einiger Tendenzen wurde bewusst auf Arbeiten in englischer, französischer und deutscher Sprache aus dem letzten Jahrzehnt beschränkt².

1 Ein älterer Rezensionsaufsatz, Hinrichs 1998, spricht bereits von den «neuen Geographien» der chinesischen Medizin. Lessell 2006 ist eine Bibliographie der westlichen Literatur zur chinesischen Medizingeschichte von 1473 bis 1900. Lee 2003 gibt eine Literaturübersicht zur florierenden taiwanesischen Medizingeschichte: http:/www.ihp.sinica.edu.tw/ medicine/ ashm/lectures/Jender\%20Lee-ft.pdf. Für eine Bibliographie der chinesischsprachigen Körpergeschichte vgl. Li/Kuriyama/Zheng 2000: http://www.ihp.sinica.edu.tw/ medicine/book/ book.htm.

2 Es ist anzunehmen, dass diese Produktion weitgehend der Etablierung und dem Ausbau von wissenschafts- und medizingeschichtlichen Instituten folgt, die sich mit dem älteren China befassen. Letztmals diskutierte Sivin 1988 die institutionelle Lage dieser Forschungsfelder. Arbeiten aus anderen als den obgenannten Sprachräumen konnten nicht berücksichtigt werden.

Dr. phil. Rudolf Pfister, SNF-Projekt «Psychophysical Techniques in Ancient and Medieval China», Universität Basel, Eggfluhstrasse 6, CH-4054 Basel (rodoX@gmx.net). 


\section{Manuskriptfunde zu gesundheitsfördernden und sexuellen Körper- techniken}

Manuskriptfunde in altchinesischen Gräbern haben die Quellenlage für den Zeitraum des 3.-2. Jahrhunderts v.u.Z. erheblich verbessert ${ }^{3}$. Gerade für die Körpertechniken wichtige Bereiche - wie die «zimmerinterne Kunst> (fáng zhōng shù) für ungleichgeschlechtliche Paare und die «Lebensnährung> (yăng shēng) der eigenen Person - waren zuvor nur noch aus den wenig aufschlussreichen Anspielungen in altchinesischen philosophischen Werken oder gar bloss über Angaben zu Werktiteln aus den Bibliographien der Dynastiegeschichten bekannt.

Damit verbunden war ein Überdenken der Periodisierung der chinesischen Medizinklassiker. Die Sammlungen klassischer heilkundlicher Traktate - unter dem Titel Innerer Klassiker des Gelben Herrschers (huáng dì nèi jīng) - gelten nun durchwegs als jünger; statt sie dem 4. Jahrhundert v.u.Z. zuzuweisen, wird ihre Zusammenstellung nun frühestens auf das 1. Jh. v.u.Z. und später angesetz $t^{4}$. Dabei gewinnt die Untersuchung ihrer stark kompilativen Struktur zwar zunehmend an Bedeutung, doch ist deren Analyse noch keineswegs abgeschlossen und dementsprechend stehen weiterhin schwierige philologische Arbeiten $a^{5}$.

In Grab Nummer 3 von Mawangdui (heutige Provinz Hunan), das 168 v.u.Z. geschlossen wurde, kamen 1973 vierzehn im weitesten Sinne heilkundliche Manuskripte auf Seide und Bambusplättchen zutage ${ }^{6}$. Die darin behandelten Themen sind überaus vielfältig und sollen im folgenden kurz und mit Angaben zu ähnlichen Funden der Zeit besprochen werden. Da hier nur die westliche Literatur zur Sprache kommt, zeigt sich ein sehr ungleichmässiger Bearbeitungsgrad der Quellen, in vielen Fällen wäre deshalb vorzugsweise die chinesische und japanische Sekundärliteratur beizuziehen, um sich ein ausgewogeneres Bild zu verschaffen ${ }^{7}$.

3 Siehe die zusammenfassende Darstellung Unschuld/Zheng 2000; englische Übersetzung 2005.

4 Vgl. Yamada 1979, 1998; Unschuld 2003.

5 Vgl.Tessenow 2002; für ein herausragendes Modell der Textgenese, vgl. Keegan 1988.

6 Für eine Gesamtübersetzung des Heilkunde-Korpus aus Mawangdui ins Englische, vgl. Harper 1998.

7 Eine Auswahlbibliographie der chinesischen Sekundärliteratur zum Heilkunde-Korpus aus Mawangdui gibt Zheng 2004. 


\section{Rezeptschriften}

Ein wichtiges Teilgebiet der heilkundlichen Literatur wird in den Rezeptschriften aus Mawangdui, Zhangjiashan (Grab 247, Grabschliessung nach 186 v.u.Z., im heutigen Hubei) und Wuwei (aus dem 1. Jh. n.u.Z., heutiges $\mathrm{Gansu}^{8}$ ) in frühen Formen greifbar. Dabei ist die Bandbreite der behandelten Themen recht gross: Eine umfangreiche Pharmazie dient beispielsweise im Mawangdui-Text Rezepturen zu zweiundfünfzig Krankheitsbildern (wŭshí'èr bìng $f \bar{a} n g^{9}$ ) der Behandlung von Hautleiden, Verletzungen wie Hunde- und Schlangenbissen, Stiche usf., gegen Krämpfe, Hämorrhoiden, Harnleiden und Hodensackschwellungen. Die Rezepturen zum Nähren des Lebens (yăng shēng $\left.f \bar{a} n g^{10}\right)$ ergänzen mit Rezepten zur Stärkung der Lebenskräfte, das heisst bei Erektionsschwäche, zur Penisvergrösserung, zur Vaginaverengung und zur sexuellen Stimulation (insbesondere mit Heiltüchern); es gibt auch Verschreibungen, um das «rasche Reisen» zu ermöglichen, gegen halbseitige Lähmungen und Schwellungen der Genitalien sowie mit Methoden zur Haarentfernung usf. Auch die Behandlungsmethoden sind vielfältig: Kauterisation, Spitzstein-Eingriff, Heissdruck, Räuchern, Baden, Massage, Schröpfen sowie magisch-rituelle Handlungen werden angewandt ${ }^{11}$.

\section{Mài-Texte und Shuangbaoshan-Figur}

Während nach der mittelalterlichen Akupunktur- und Moxa-Behandlung der menschliche Leib ein Zwölfersystem von Leitbahnen (Meridianen) aufweisen soll, sind in Mawangdui und Zhangjiashan mehrere Schriften aufgetaucht, die elf untereinander jeweils leicht abweichende 〈Aderflüsse〉 (mài) samt den ihnen zugeordneten Krankheitsbildern auflisten ${ }^{12}$. Diese Leitbahnbeschreibungen können mit dem in Shuangbaoshan (Grab 2, heutiges Sichuan, West-Han-Dynastie) zutage geförderten schwarzen Lackmodell eines Menschen verglichen werden, das mit roten Linien überzogen ist. Es erweitert zwar die Kenntnisse über frühe Entwicklungen dieser bis heute prägenden Sichtweise über die innerkörperlichen Vernetzungen und Trans-

8 Die Wuwei-Funde sind in der westlichen Literatur nur wenig behandelt worden. Siehe die Diskussion von Einzelaspekten in Xie 2005, 79-83 und Yates 2005/2006.

9 Titel des Manuskripts auf Seide durch Herausgeber. Vgl. Harper 1998, 221-304.

10 Vgl. Harper 1998, 328-362.

11 Einen Versuch, die Frühgeschichte von Akupunktur und Moxibustion aufzuzeigen, macht Yamada 1998,1-88.

12 Vgl. Harper 1998, 187-212. Lo 1998, eine noch unveröffentlichte Dissertation, enthält englische Übersetzungen der beiden Zhangjiashan-Schriften Über die Adern (mài shü) und Über das Dehnen (y̆n shü). 
porte, konfrontiert einen jedoch gleichzeitig mit der womöglich unbeantwortbaren Frage nach der intendierten Bedeutung der aufgemalten Linienführung ${ }^{13}$.

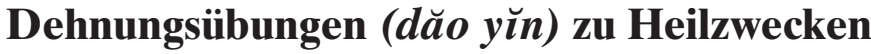

Durch besondere Formen von Turnübungen sollte das innerkörperliche 〈Fliessgeschehen〉 (qì) über die Dehnung von Muskelsträngen im Leibe in Fluss gebracht werden. Dazu fand sich in Mawangdui eine farbige Malerei auf Seide, die Illustrationen über das Leiten und Dehnen [des Fliessgeschehens] (dăo y̆n tú), die einmal 44 derartige Übungen abbildete. In Zhangjiashan behandelt eine Über das Dehnen (y̆n shü) betitelte Schrift auf Bambusplättchen eingehend gymnastische Dehnungsübungen, mit denen Krankheiten wie Erschöpfungszustände, Nacken-, Knie-, Knöchel-, Muskel- oder Rückenschmerzen und vieles mehr angegangen werden ${ }^{14}$.

\section{Sexuelle Körpertechniken}

Unter die Techniken zur 〈Lebensnährung〉 (yăng shēng ${ }^{15}$ ) fallen neben heilgymnastischen Übungen und besonderen Ernährungsvorschriften auch die sexuellen Körpertechniken. Drei Texte aus dem Heilkunde-Korpus von Mawangdui beinhalten neben den oben bereits angetönten, entweder eher krankheitsbezogenen oder aber stärkenden und stimulierenden Massnahmen aus den Rezeptschriften auch gezielt auf den Mann ausgerichtete Ausführungen ${ }^{16}$. Letztere dienen der Anleitung, um eine über den sexuellen Kontakt mit der Frau ausgelöste besondere Bewusstseinserfahrung herbeizuführen. Dabei lässt sich die Frau von den Zuckungen der Lust tragen, während der Mann sie optimal zu stimulieren versucht, ohne aber selbst muskuläre Spannung aufzubauen. Die drei Manuskripte bieten überaus reich-

13 Zum Vergleich der Bahnen, vgl. He/Lo 1996; siehe auch Engelhardt 1998a. Besonders zur Schmerzleitung, vgl. Lo 1999; Hsu 2005.

14 Harper 1998, 310-327, mit einigen schwarzweissen Umzeichnungen; Lo 1998, 342-355; Engelhardt 1998b, 2001a (summarische Inhaltsübersicht). Zur frühen Heilgymnastik, vgl. Despeux 2004.

15 Zur «Lebensnährung», vgl. Lo 1998, 2001;im mittelalterlichen China: Stein 1999. Kritisch zum Begriff der Makrobiotik im chinesischen Kontext: Collins/Kerr 2001.

16 Übersetzungen der drei Texte auf Bambusplättchen - (a) Zehn Befragungen (shí wèn), (b) Über das Vereinen von Yin und Yang (hé yīn yáng) und (c) Diskussion der optimalen Methode unter den Himmeln (tiān xià zhì dào tán) - geben Harper 1998, 385-438 (a)-(c); Wile 1992, 77-83, nur Texte (b)-(c); Pfister 2003, nur Texte (b)-(c); Pfister 2008 (a)-(c). 
haltige Details zur Physiologie von Frau und Mann, zum Ablauf der sexuellen Begegnung und zu postulierten Prozessen im Körperinneren.

Der Vermeidung von Emission und Ejakulation des Mannes gilt dabei ganz besondere Aufmerksamkeit, während alles Notwendige unternommen sein will, um den Erguss der Frau (d.h. die weibliche Ejakulation) zu fördern. Dazu gehören nicht nur die Variation von Stellungen, Häufigkeit, Geschwindigkeit, Richtung und Art der Penetration, sondern auch die Betonung der Langsamkeit und der geschickten Antwort auf den jeweiligen Erregungszustand der Frau. Währenddessen versucht der Mann, seinen Atemgang gleichmässig zu halten und bei Ejakulationsdrang die Stossbewegung zu verlangsamen, das Glied leicht zurückzuziehen, um nicht seinen lebenswichtigen Saft, den 〈Seim〉 oder die 〈Essenz〉 (jīng), zu verlieren ${ }^{17}$. Dies bewirkt bei ihm nach einem neunfachen Durchgang eine ganzkörperliche Entspannung und Lichtdurchflutung - die 〈geistige Erhellung〉 (shén míng)-, ein euphorisch-überwältigendes Erlebnis, das zu erlangen, die Männerwelt mit grossem Eifer trachtet. Dieses kommt nicht ohne die gehörige Stimulation der Frau zu mehrfachem Erguss zustande, denn nur so glaubt man zu gewährleisten, dass ihr 〈Seim〉 den seinen nährt und so seine Lebensprozesse in Schwung gebracht und eine lange Lebensspanne möglich wird ${ }^{18}$.

Ausgehend von diesem reichhaltigen Material, können nun die von Robert van Gulik vorgegebenen Ansätze zu den mittelalterlichen sexualtechnischen Texten ergänzt, korrigiert, verändert und präzisiert werden ${ }^{19}$. Dies geschieht nicht nur hinsichtlich von zahlreichen Details, sondern insbesondere durch die Ausarbeitung von geschlechtsspezifischen und religiösen Aspekten der <zimmerinternen Kunst ${ }^{20}$, durch die Darstellung gleichgeschlechtlicher Sexualität ${ }^{21}$ und von medizingeschichtlichen Gebieten, insbesondere der Konzeption innerer Prozesse beim $\mathrm{Mann}^{22}$, sowie durch die Relektüre auch des klassischen Schrifttums im Hinblick auf sexuelle Motive ${ }^{23}$.

17 Darstellungen der Mawangdui-Sexualtechniken und -Texte geben: Harper 1987, 1998 (Prolegomena), 2005a (Aphrodisiaca); Li/McMahon 1992; Pfister 1997, 2002, 2006a, 2007, 2008. Zur Sprache des Sexuellen, vgl. Middendorf 2007, die Sprachfiguren der Mawangdui-Texte mit denjenigen der Oden (shī jīng) in Beziehung setzt. Zum Liebszauber, vgl. Liu 2005.

18 Vgl. Pfister 2006a, 2008.

19 Gemeint sind die kürzlich nachgedruckten Werke: Van Gulik [1951] 2004 und [1961] 2003. Siehe die kritischen Kommentare zu diesen Arbeiten: Furth 1994, 2005; Hinsch 2005.

20 Umekawa 2004, 2005.

21 Hinsch 1990, 2005.

22 Pfister 2006b, 2008.

23 Goldin 1999, 2001, 2002, 2006; Riegel JK 1997; Middendorf 2007. 


\section{Geburtshilfe und -brauchtum}

Das quadratische Seidenmanuskript Abhandlung über Fötus und Geburt (tāi chăn shū) bringt in der oberen Hälfte zwei Diagramme: Dabei handelt es sich beim Diagramm in der rechten oberen Hälfte um zwei menschliche Figuren, die mit den Namen der zwölf «[Erd-]Zweige $(z h \bar{\imath})$ beschriftet sind und die wohl die Situation für die Jahreszeiten Frühling/Sommer bzw. Herbst/Winter aufzeigen ${ }^{24}$. Je nach Geburtszeit kann das Schicksal des Neugeborenen aus Erdzweig und Jahreszeit ermittelt werden ${ }^{25}$. In der linken oberen Hälfte illustriert eine quadratische Kartierung die Methode zum 〈Verwahren der Nachgeburt〉 (cáng $b \bar{a} o)^{26}$. Der Textteil der Schrift befasst sich mit der Entwicklung der Leibesfrucht während zehn Monaten und der jeweils geeigneten Nahrung für die Schwangere.

\section{Medizinische Gerätschaften}

Es bleibt Desiderat, die materiellen Relikte der heilkundlichen Tätigkeiten zu erforschen; lediglich über die Antike liegen einigen Untersuchungen zu Nadeln, Jade- und Steingerät vor ${ }^{27}$.

\section{Quellenïbersetzungen}

Medizinhistorisch und philologisch überzeugende Übersetzungen von medizin- und körpergeschichtlichen Quellen sind (ebenso wie deren kritische Edition) weiterhin dünn gesät. Nebst den bereits erwähnten Arbeiten zu frühen Manuskriptfunden sollen deshalb einige Beiträge zur Sprache kommen, die längere Teile oder ganze medizinische Texte übersetzen ${ }^{28}$.

24 Bei den 〈[Erd-]Zweigen〉 $(z h \bar{l})$ handelt es sich um eine Nomenklatur für Zeitabläufe, die mit den 〈[Himmels-]Stämmen〉 $(g \bar{a} n)$ kombiniert einem Sexagesimalsystem folgen. Vergleiche unten die Diskussion der Iatromantie.

25 Vgl. Harper 1998, 372-384; schwarzweisse Umzeichnungen der Diagramme, S. 373, 376-377; Riegel A-M 1999, 53-58 (auszugsweise deutsche Übersetzung).

26 Der Kolloquiumsbericht Sartorius 2004 sammelt Beiträge zum Brauch der Nachgeburtsbestattung in verschiedenen Kulturen und Zeiten. Leider ist der Kurzbeitrag zum alten China (Riegel A-M 2004), der sich auf den Mawangdui-Text bezieht, nicht bloss nachlässig formuliert, sondern auch sachlich wenig kompetent.

27 Yamada 1998,1-88; Lo 2002. Zumeist (spät-)kaiserzeitliche Materialien behandelt Unschuld 1995.

28 Siehe unten auch die Diskussion von Flohr 2001 (Kinderheilkunde) und Kovacs/Unschuld 1998 (Augenheilkunde). 
Sima Qians Aufzeichnungen des Geschichtsschreibers (sh $\breve{\jmath}$ ji) enthalten im zweiten Teil von Kapitel 105, Biographien von Bian Que und Cang gong (etwa 90 v.u.Z.), ein Memorandum des Arztes Chunyu Yi mit fünfundzwanzig medizinischen Fallgeschichten, das von ihm wohl nach 164 und vor 153 v.u.Z. verfasst worden sein dürfte. Diese für die frühe Kaiserzeit einmalige Dokumentation bildet den Kern der Übersetzung und einer Reihe von Studien von Elisabeth Hsu, die sich mit Themen wie der frühen Pulsdiagnostik, den Auffassungen der Krankheit und des heilkundlichen Argumentierens sowie der Rolle des Tastsinns befassen. Dabei zeigt sich die Eigenart des medizinischen Ansatzes von Chunyu Yi ebenso deutlich im Vergleich mit den GrabManuskripten wie auch mit den späteren Puls- und Medizinklassikern ${ }^{29}$.

Paul Unschuld hat, in Vorwegnahme der Publikation der Gesamtübersetzung, eine vertiefte Darstellung und Inhaltsanalyse der - wohl in der frühen Kaiserzeit - zum Medizinklassiker Innerer Kanon des Gelben Herrschers: Grundlegende Fragen (huáng dì nèi jīng sù wèn) zusammengefügten heilkundlichen Traktate geliefert. Darin werden die Textgeschichte, die YinYang- und Fünf-Phasen-Theorien, die Auffassungen über den menschlichen Leib, über $Q i$ und Blut, Krankheitsbilder wie Wind, Malaria, Erkältung etc., die Diagnostik derselben u.v.m. detailreich erläutert ${ }^{30}$.

Ein Autorenkollektiv hat sich mit der Zusammenstellung und Übersetzung der Zeugnisse zu einem einzelnen Krankheitsbild - dem Husten auseinandergesetzt. Die Arbeit diskutiert an diesem Beispiel die mittelalterliche Konzeption der Krankheit, die zugehörigen Behandlungsstrategien und übersetzt mehr als 130 Rezepturen, die vor dem 8. Jahrhundert dagegen empfohlen wurden ${ }^{31}$.

Sun Simiao (581-682, Tang) widmet drei Kapitel der Tausendfach goldwerten wichtigen Verschreibungen für dringliche Fälle (bèi jí qiān jīn yào fāng, um 652) den Verschreibungen für Frauen, die Sabine Wilms nun ins Englische übersetzt hat. Er ist damit der erste Autor, der ausdrücklich die Notwendigkeit betont, dass Frauenleiden 〈separater Verschreibungen〉 (bié fāng) bedürften ${ }^{32}$.

29 Hsu 2001a (mit Übersetzung); vgl. Hsu 2001c, 2005 (Tastsinn).

30 Unschuld 2003.

31 Despeux/Obringer 1997.

32 Wilms 2002. Für eine Zusammenfassung mit Fokus auf die Menstruation, vgl. Wilms $2005 a$. Zu Sun Simiaos Quellen zu den Kapiteln über Frauenkrankheiten, vgl. Wilms 2006b. 


\section{Dunhuang-Manuskripte - Zeugnisse heilkundlicher Vielfalt}

Eine zweite grosse Cache von Texten auf Papier kommt aus Grotte 17 in Dunhuang (Provinz Gansu). Tausende von mittelalterlichen Manuskripten lagerten dort bis sie Anfang des 20. Jahrhunderts wiederentdeckt wurden. Erst vor kurzem lancierte man Projekte zur Aufarbeitung dieses Schatzes. Das International Dunhuang Project digitalisiert die Manuskripte und publiziert sie im Internet ${ }^{33}$. In Divination et société dans la Chine médiévale findet man Einführungstexte zu den Einzelmanuskripten und eine thematische Quellenbeschreibung über die Wahrsagetexte im allgemeinen ${ }^{34}$, insbesondere aber zu den mehr oder weniger heilkundebezogenen Gebieten der Iatromantie ${ }^{35}$, Physiognomie ${ }^{36}$, Traumdeutung ${ }^{37}$, Hemerologie $^{38}$, Auguromantie ${ }^{39}$, Kleromantie $^{40}$ und des Talismangebrauchs ${ }^{41}$. Ein Folgeband zu medizinischen und medizinisch-religiösen Texten ist in Arbeit ${ }^{42}$. Er wird eine Aufsatzsammlung ergänzen, worin bereits eine Anzahl heilkundlicher Quellen aus Dunhuang knapp charakterisiert wurde ${ }^{43}$, die jedoch zusätzlich weitere Aspekte populärer Heiltraditionen wie die Schaubilder von Moxibustions-Stellen ${ }^{44}$, Diätanweisungen ${ }^{45}$, Pharmakologie ${ }^{46}$, Wind-Krankheiten ${ }^{47}$ und Liebeszauber ${ }^{48}$ behandelt. Michel Strickmann seinerseits lotet die daoistischen und buddhistischen Texte über Krankheit und religiöses Gesetz, Exorzismen, Besessenheit, Zaubersprüche u.v.m. aus. Des weiteren diskutiert er die bemerkenswerte Technik der Ensigillation - wobei mit Siegeln bedrucktes Papier zu Asche verbrannt und geschluckt wird; zusammen mit Visualisationen, Beschwörungen, besonderen Körperhaltungen und Handgesten soll das die Gesundheit fördern oder Krankheiten überwinden helfen ${ }^{49}$.

33 Frei zugänglich unter http://idp.bl.uk.

34 Kalinowski 2003; vgl. Kalinowski 2005.

35 Siehe dazu unten den Abschnitt zur Innovation in der chinesischen Medizin (Harper-Studien).

36 Vgl. Despeux 2003b, 2005a.

37 Drège/Drettas 2003.

38 Kalinowski 2003a.

39 Despeux 2003a.

40 Kalinowski 2003b.

41 Mollier 2003.

42 Projekttitel: «Médicine, religion et société dans la Chine médiévale. Etude des manuscrits de Dunhuang de la Bibliothèque Nationale de France et de la British Library».

43 Lo/Cullen 2005. Darin der Überblick über die medizinischen Manuskripte, Wang 2005a, und eine Quellenbeschreibung, Wang 2005b.

44 Lo 2005.

45 Sakade 2005; Wang 2005d (Dekokte). Vergleiche Engelhardt 2001b.

46 Wang 2005c; Mayanagi 2005.

47 Chen 2005.

48 Liu 2005.

49 Strickmann 2002. Siehe die Besprechungen von Robert Duquenne in Cahiers d'Extrême-Asie 14 (2004) 423-440 und Henrik H. Sørensen in History of Religions 43.4 (2004) 319-332. 
Die Pluralität der mittelalterlichen Ansätze, die zwischen Diagnose und Prognose von krankhaften Prozessen oszillieren ${ }^{50}$, deren Einbettung in religiöse Gemeinschaften und deren Bezüge und Konkurrenzen zur Gelehrtenmedizin und zur kaiserlich geförderten Medizin wird zweifellos noch in eine eingehendere und die Einzelarbeiten synthetisierende Darstellung münden. Das von offizieller Seite immer wieder Ausgesonderte, vielleicht auch das Überholte und Verworfene, kann in den Dunhuang-Manuskripten ansatzweise und dazu in einem frühen Zustand wieder zu heben und in den Diskurs der Geschichtsschreibung aufzunehmen versucht werden.

\section{Innovation in der chinesischen Medizin}

Zum Gedenken an Lu Gwei-djen (1904-1991), Joseph Needhams Mitarbeiterin und zweite Ehefrau, befasste sich 1995 ein Workshop in Cambridge mit der Frage der Innovation in der chinesischen Medizin ${ }^{51}$. Dies ist gewiss auch als Reaktion auf die in der Öffentlichkeit und in populären Schriften hartnäckig beschworene vieltausendjährige Tradition der Traditionellen Chinesischen Medizin (TCM) zu verstehen, die die Geschichtlichkeit auch in diesem Bereich des menschlichen Handelns nicht sehen will. An dieser Stelle soll nur kurz auf die Beiträge der Festschrift hingewiesen werden, die die Frage der Innovation in älterer Zeit angehen.

Vivienne Lo versucht, den Einfluss der Kultur der «Lebensnährung〉 in der frühen Kaiserzeit (West-Han-Dynastie) auf die medizinischen Traktate über Moxibustion und Akupunktur nachzuzeichnen. Sie findet, dass bei der topographischen Beschreibung der Leibesoberfläche in sexualtechnischen Texten verwendete Begriffe auch Eingang in die Namensgebung der «acumoxa points» - gemeint sind geringfügige Vertiefungen in den als «Gräben» aufgefassten 〈Aderflüssen〉 (mài) - fanden. Typischerweise handle es sich dabei auch um einen sprachlichen Transfer vom Eigenerleben und Versuch, die eigene Gesundheit zu verbessern, hin zur Ausrichtung auf Patienten und Patientinnen, wobei diese Beobachterperspektive auch mit der Reduktion auf visuelle Aspekte einhergehe ${ }^{52}$.

50 Vgl. Despeux 2005a.

51 Vgl. Hsu ed. 2001b. Als Zeugnis der Zusammenarbeit von Lu Gwei-djen und Joseph Needham (1900-1995) ist von Nathan Sivin posthum eine unabgeschlossene Geschichte der chinesischen Medizin mit älteren Beiträgen als Band VI.6 von Science and Civilisation in China herausgegeben worden (Needham/Lu 2000).

52 Lo 2001. Zum Beitrag Hsu 2001c siehe oben im Abschnitt über die Fallgeschichten des Chunyu Yi. 
Donald Harper versteht die Kunst der Voraussage von gesundheitsrelevanten Ereignissen oder Iatromantie als eine mögliche Vorläuferin der korrelativen Kosmologie, die die Han-Eliten dominiert habe. Evidenzen des 4. und 3. Jahrhunderts v.u.Z. werden mit einer Prognose des Chunyu Yi (2. Jh. v.u.Z.) und späteren Prognosen aus der Wirkmächtigen Achse (ling $s h \bar{u}$ ) kontrastiert, um zu zeigen, dass die im Baoshan-Wahrsagetext (für die Jahre 317-316 v.u.Z.) benutzten sexagesimalen Zeit-Zyklen (eine Kombination der zehn Himmelsstämme und zwölf Erdzweige) von hemerologischen auf heilkundliche Thematiken übertragen und mit korrelativen Systemen wie den 〈Fünf Phasen $>$ (wŭ xíng) und Yin-Yang-Kontrasten kombiniert wurden ${ }^{53}$.

Ute Engelhardt sieht in Sun Simiaos Kapitel Über diätetische Behandlung (shí zhi) in den Tausendfach goldwerten wichtigen Verschreibungen für dringliche Fälle (bèi jí qiān jīn yào fāng) die erste erhaltene materia dietica, die auch den theoretischen Rahmen für das darauf Folgende absteckt. Suns Student Meng Shen (621-713) schreibt das erste vollständig der Diätetik gewidmete Buch, die nicht mehr erhaltenen Rezepturen zum Ergänzen und Nähren (bŭ yăng fāng), und dessen Werk wiederum ergänzt sein Student Zhang Ding (8. Jh. n.u.Z.) mit der Materia medica der diätetischen Therapie (shí liáo běn căo, zwischen 721-739), die als erste nur die <diätetische Therapie> (shí liáo) behandelt und die praktischen Anwendungsmöglichkeiten betont $t^{54}$.

\section{Innovation in der Song-Dynastie (960-1279)}

Da sich mehrere Arbeiten mit medizinischem Wandel und Innovation in der Song-Dynastie auseinandersetzen, stellt sich diese Periode als die am weitesten erforschte unseres Berichtszeitraumes dar.

Frédéric Obringer erörtert eine «nicht-radikale Innovation» (im Sinne François Sigauts): Die Verwendung von Arsen-Derivaten hat eine lange Geschichte in China, beispielsweise benutzt der Mawangdui-Text Rezepturen zu zweiundfünfzig Krankheitsbildern Realgar $\left(\mathrm{As}_{2} \mathrm{~S}_{2}\right)$ gegen Hautbeschwerden und äusserlichen Parasitenbefall. Eine Neuordnung der Begrifflichkeit begleitet die technische Innovation des Erhitzens von Arsenkies, um Weissarsenik oder glasigen Arsenolith (Arsenblüte, $\mathrm{As}_{2} \mathrm{O}_{3}, p \bar{\imath}$ shuāng) zu gewin-

53 Harper 2001. Zur Iatromantie siehe auch Harper 2003, 2005b. Für eine ausführliche Darstellung und Übersetzung des Textes mit den Wahrsagungen zur Krankheit von Shao Tuo, dem Grabherrn von Baoshan Grab M2 (Grabschliessung 316 v.u.Z.), vgl. Cook 2006, 19-42, 153-210.

54 Engelhardt 2001b. 
nen. Dies stellt gegen Ende des 10. Jahrhunderts eine Verfahrensneuerung dar. Sie bringt ein synthetisches und nach festgelegter Methode hergestelltes Produkt neu in den Heilmittelschatz ein. P̄ shuāng wird besonders gegen Wechselfieber in oft gefährlich hohen Dosen (geschätzte 80-100 mg als tägliche Dosis) eingesetzt. Durchfall und Erbrechen wird als mögliche Wirkung dieses 〈Attackierens der Hitze mit Hitze〉 (y̆ rè gōng rè) beschrieben. Folglich gerät das Produkt nach etwa zweihundert Jahren in Diskredit: Die Droge verliert das Prestige, das sie während der Song-Dynastie zunächst gewonnen hat, und wird zunehmend weniger verschrieben. Ihre Toxizität ist von Anfang an bekannt, doch die Bestimmung und Klassifikation der diversen Grundmaterialien stellt die Anwender ebenso vor Probleme wie die Instabilität des Arsen-Anhydrids, so dass mögliche kurative Vorteile durch die Giftigkeit in Frage gestellt wurden ${ }^{55}$.

Catherine Despeux beschreibt ein prognostisches System, das zunächst aussermedizinisch gebraucht ab etwa dem 10. Jahrhundert auch für medizinische Zwecke adaptiert wird. Bei dem System der sünf zirkulatorischen Phasen und sechs jahreszeitlichen Einflüsse> (wŭ yùn liù qì) geht es darum, über komplexe Berechnungen und deren Interpretation Kenntnisse zu erlangen, die über das Oberflächliche hinausgehen, sie mit observierbaren Tatsachen zu vergleichen und Schlüsse auf den Krankheitsverlauf zu ziehen. Despeux versichert, dass das System nur beschränkt anwendbar gewesen sei, besonders im Zusammenhang mit der Prognose, Verhinderung und Behandlung von epidemischen Krankheiten und für prophylaktische und therapeutische Zwecke. Die Komplexität der korrelierten Elemente ermöglichte es, innovative Aspekte ohne Bruch mit früheren Lehren einzuführen, was vielleicht auch darauf zurückzuführen ist, dass dieses System besonders vom Kaiserhof gefördert wurde ${ }^{56}$.

Asaf Moshe Goldschmidts Dissertation versucht, medizinischen Wandel in der Nord-Song-Dynastie (960-1127) aus gesellschaftlichen Verhältnissen zu erklären ${ }^{57}$. Die Reaktion des Kaiserhauses auf die Epidemien (besonders zwischen 1045 und 1060), die Verschiebung der Bevölkerungsmehrheit in den Süden und die Verdoppelung der Bevölkerung auf über 100 Millionen Einwohner seien Faktoren, die sich auf drei von Goldschmidt konstruierte medizinische Bereiche ausgewirkt hätten: (a) auf die «eklektische Praktik» der Drogentherapie; (b) auf die «klassische Medizin» der Akupunktur-MoxaBehandlung (Aku-Moxa-Therapie) und (c) auf die Bekämpfung der Epidemien unter dem Titel einer Neubewertung der «Cold Damage Disorders〉

55 Obringer 2001. Zur Thematik der Giftstoffe, vgl. Obringer 1997.

56 Despeux 2001.

57 Goldschmidt 1999. Siehe auch Goldschmidt 2001, 2005. 
(shāng hán, wörtlich etwa «schädigende Kälte»). Während letztere in der Heilkunde vor der Nord-Song-Dynastie noch vernachlässigt worden seien, erhielten sie mit der zunehmenden Besiedelung und Bevölkerungsdichte im Süden (Jiangnan) vermehrte Bedeutung im medizinischen Diskurs. Es handle sich dabei um akute infektiöse Fiebererkrankungen von der gewöhnlichen Erkältung, Influenza, bis hin zu typhoiden Fiebern und hochansteckenden Erkrankungen. In die Aku-Moxa-Therapie wurden zunehmend anatomische Kenntnisse eingebaut, und zu Lehrzwecken goss man lebensgrosse Bronzefiguren mit oberflächlich markierten Akupunkturstellen; damit erreichte man nunmehr eine standardisierte Topographie der Oberfläche des menschlichen Leibes. Das kaiserliche Erziehungssystem wurde ausgebaut und auf die «klassische Medizin» ausgedehnt. In der Drogentherapie kam es zu einer Integration von Rezepturen mit nur einem Heilmittel und Rezeptformeln, die Eigenschaften der Einzeldrogen wurden vertieft untersucht und eine kaiserliche Pharmazie wurde eingerichtet.

T. J. Hinrichs spinnt den Faden Goldschmidts fort; denselben Zeitraum behandelnd, zeigt sie, «knitted into a spiral», die Differenzen der Haltungen von Schamanen des Südens und ihrer Klientele im Verhältnis zu Gelehrtenärzten und Beamten aus dem Norden Chinas, die apotropäischen und exorzistischen Heilmethoden des Südens und das Vermeiden von erkrankten Personen während Epidemien im Kontrast zu den offiziell vertriebenen Heilmitteln und erzieherischen Massnahmen der kaiserlichen Regierung aus dem Norden. Unter letztere kann man das Auspeitschen von Schamanen und das Zerstören ihrer Schreine ebenso zählen wie die Produktion und den Vertrieb medizinischer Texte und Medikamente. Die Eigenarten des Klimas und der Bevölkerung des Südens stellten eine Herausforderung an die Elite aus dem Norden dar, die sich jedoch selbst und ihre neue Umgebung zunehmend veränderte ${ }^{58}$.

\section{Frauenheilkunde und Geburtshilfe}

Das Thema der Frau in der Heilkunde ist ein vielbeachtetes ${ }^{59}$. Charlotte Furth verfolgt mit ihrem Buch über die chinesische Medizin von 960 bis 1665 das dreifache Ziel, (a) eine Medizingeschichte der gewählten Periode zu schreiben, (b) das Thema der Geschlechtsrolle in dieser Geschichte zu thematisie-

58 Hinrichs 2003.

59 Furth 2005/2006 ist eine Bibliographie der Sekundärliteratur zum Thema Medizin und Gender im frühkaiserzeitlichen China. Zu Medizin und Gender in der Tang-Dynastie, vgl. Lee 2003. 
ren und (c) eine Diskursanalyse zu geben, die den medizinischen und den sozialen Körper der Frau in Beziehung setze. Furths drei Hauptthesen entlang dieser Zielsetzungen sind: (1.) Die Gynäkologie (fükē) wird während der Song-Dynastie zu einer ausgeprägten Abteilung des heilkundlichen Wissens und betont bei der Behandlung von Frauen insbesondere «the leadership of Blood». Mit dem grossgeschriebenen Blood (xuè) sei die Gesamtheit der Blutaspekte angesprochen, in seinen verschiedenartigen Manifestationen als Menstruation, Muttermilch oder Blut im Leibe. (2.) In Absetzung zur Behauptung eines eingeschlechtlichen Modells für die «klassische europäische Medizin» (nach Thomas Lacqueur) versucht Furth, den «Körper der klassischen chinesischen Imagination» als «ursprünglich androgyn» erscheinen zu lassen, was eine durchaus problematische These abgibt. (3.) Schliesslich vereinfacht Furth die über Jahrhunderte kompilierten Sammlungen von Traktaten in der Tradition des Inneren Klassikers des Gelben Herrschers (huáng dì nèi jīng) zu einem einheitlichen Konstrukt, das sie «the Yellow Emperor's Body» nennt ${ }^{60}$.

Durch Furths Buch inspiriert, spinnen die Autorinnen und Autoren der Aufsatzsammlung Medicine for Women in Imperial China den Faden für die Periode vor der Song-Dynastie weiter ${ }^{61}$. Robin D. S. Yates konstatiert für die frühkaiserzeitliche Frauenmedizin, dass darin religiöse Rituale eine wichtige Rolle spielten: Daoistische, buddhistische und volksreligiöse Praktiken hätten die Schwangerschaft, die Geburtshilfe, das Kindbett usf. begleitet. Yates setzt zu Recht den Beginn von geschlechtsspezifischen medizinischen Teilgebieten früh in die Han-Dynastie oder noch früher. Akzentuiert habe sich eine eigentliche Frauenmedizin jedoch erst in der Sui- und frühen Tang-Dynastie, und im Schrifttum sei auch vermehrt über Geschlechtsdifferenzen theoretisiert worden. Die Situation bleibe aufgrund der lückenhaften Quellenlage indessen schwer beurteilbar. Die Gesamtheit der pluralistischen, reichhaltigen und komplexen Traditionslinien bilde die Basis der Verdichtung zu einer Gynäkologie in der Song-Dynastie und danach ${ }^{62}$.

Sabine Wilms entwickelt anhand von mittelalterlichen Texten eine sich von Furths Androgynitäts-These abhebende Darstellung, wonach die klinischen Befunde und Körperwirklichkeiten der spezifisch weiblichen Prozesse der Fortpflanzung und der Schwangerschaft die Heilkundigen nachgerade dazu gezwungen hätten, «to look beyond the androgynous ideal» und zu rea-

60 Furth 1999.

61 Leung 2005/2006. Siehe auch meinen Rezensionsaufsatz: Pfister, Rudolf, «Über Frauenheilkunde im mittelalterlichen China: Idealisierte die Medizin ein androgynes Körperbild oder nicht? Beherrscht darin der Blutaspekt die Frau? Wann entstand eine Heilkunde speziell für Frauen?», Asiatische Studien - Etudes Asiatiques 61.3 (2007) 989-1006.

62 Yates 2005/2006. 
lisieren, dass Frauenleiden kategoriale Unterschiede von Männerleiden aufwiesen, was zur Geburt der Gynäkologie als eigenständigem medizinischem Gebiet führte. Wilms beschliesst ihren Beitrag mit der Darstellung der Betonung des Blutes (Furths erste These). Ihr zufolge stellt die Erkenntnis, dass Blut die grundlegende Lebensflüssigkeit des weiblichen Körpers sei, die zentrale medizinische Innovation in der Entwicklung der chinesischen Gynäkologie dar. Dies markiere eine «faszinierende Abkehr» von der damals geltenden Orthodoxie, wonach der zentrale Platz $Q i$ eingeräumt werde, das «material basis and life-sustaining force of all existence» sei. Die Entdeckung von $Q i$ stelle für sich genommen die wichtigste Entwicklung der chinesischen Medizin dar und habe bis heute unangefochten Bestand ${ }^{63}$.

Lee Jen-der stellt detailreich Zeugnisse zur Kindsgeburt zusammen ${ }^{64}$. Geburten stellen eine Gefährdung für das Leben der Frau dar, und folglich suchte man auch im alten China nach Wegen und Möglichkeiten, um dabei Risiken auszuschalten. Besondere Sorgfalt liess man der Schwangeren angedeihen, die 〈[vor der Niederkunft] in den Monat einging ( rù yuè): Es werden Rezeptvorschriften gegeben, die 〈den Fötus glitschig machen〉 (huá tāi) sollen. Ausserdem bereitete man einen besonderen Ort entweder innerhalb oder ausserhalb des Hauses vor, indem man eine 〈Geburts-Hütte〉 (chăn lú) respektive ein 〈Geburts-Zelt〉 (chăn zhàng) errichtete. Diese Struktur wurde nach besonderen 〈Geburts-Karten〉 (chăn tú) im Raum orientiert; entweder separat oder später zu einer Karte kombiniert, gaben sie Auskunft (a) über die Orientierung des Zeltes bzw. der Hütte, (b) über die Himmelsrichtung, nach der sich die Frau auszurichten hatte, und (c) wo die Plazenta vergraben werden sollte. Die Niederkunft selbst ist nicht kalkulierbar und gibt Anlass zu Sorge und Furcht. Die Kreissende wird angewiesen, entweder eine sitzende oder supine Haltung einzunehmen. «Frauen gehen zu Boden und hocken sich auf Stroh als erwarteten sie den Tod», heisst es in Chen Yanzhis Rezeptvorschriften mit Heilmitteln der minderen Kategorie (xiăo pŭn fäng) aus dem 5. Jahrhundert u.Z. Damit sie sich auf etwas lehnen oder stützen kann, hängt man Seile, Tücher oder Stangen auf oder hält sie an der Hüfte. Stroh und weiches Material wird ausgelegt; mancherorts gibt es auch Geburtskörbe, die das Neugeborene aufnehmen sollen. Die Heilkundigen raten davon ab, zuviel Aufruhr um die Kreissende zu veranstalten und wollen so der Natur freien Lauf lassen und unnötige Störungen vermeiden. Treten Komplikationen auf, so gibt es allerhand pflanzliche Mittel, die die Geburt beschleunigen sollen. Nicht alle Ingredienzien der medizinisch genutzten Pflanzen stehen allerorten zur Verfügung, und so behilft man sich mit ande-

64 Lee 2005/2006, vgl. zum Ammenwesen Lee 2000. 
ren Behandlungen, wie dem Erwirken von Erbrechen oder Niesen, man gibt besondere Massagen oder versucht, das Kind wieder zurück in den Schoss zu bringen, um so erneut den Geburtsprozess einzuleiten. Nach der Niederkunft gilt es, die 〈Postpartum-Erkrankungen〉 (chăn hòu bìng) zu behandeln: Man verbrennt das unterlegte Stroh; um die emotionale Balance der Mutter zu wahren, darf sie sich nicht selbst betrachten; man fragt nicht nach dem Geschlecht des Kindes, weil die Antwort womöglich nicht ihren Erwartungen (auf einen männlichen Nachkommen) entspräche. Besonders die BlutSchwindel und Konvulsionen der Mutter müssen mit Herbalmedizin behandelt werden. Bewusstlose werden mit kaltem Wasser, Essig oder Likör zu wecken versucht. Drei Tage Bettruhe, gefolgt von Beobachtung bis zum siebten Tag nach der Geburt, sollten sicherstellen, dass nicht eine lebensbedrohende Krankheit aufkommt. Es fällt schwer, die soziale Bedeutung einer Geburt anhand der spärlichen Quellen abzuschätzen. Die Bedeutung des Gatten für direkte oder vermittelte Hilfestellungen bei der Geburt, sein Einfluss auf Entscheidungen über Leben und Tod der Mutter oder des Kindes sind nicht systematisch überliefert. Zumindest unter gewöhnlichen Leuten dürfte jedoch der Hausvorstand und werdende Vater einen nicht unwesentlichen Beitrag zu leisten gehabt haben. Ökonomische Unterschiede werden die Möglichkeiten zur Vorbereitung der Geburt, zu ihrer Unterstützung durch Geburtshelferinnen und bei Komplikationen das Zuziehen von Heilkundigen aller Art bis hin zum männlichen Arzt beeinflusst haben ${ }^{65}$.

\section{Spezialitätenmedizin}

Im Zuge der Auffächerung der Forschung sind auch einige Spezialgebiete der Heilkunde untersucht worden, wodurch sich die Tendenz vertieft, von einer allzu vereinheitlichenden, vereinfachenden und übersystematisierenden Betrachtung der Vergangenheit abzurücken.

\section{Kinderheilkunde}

Dank seiner dreiteiligen Schrift Direkte Unterweisungen über die Arzneien für Kleinkinder und über deren Krankheitsbilder (xiăo ér yào zhèng zhí jué),

65 Ergänzende Materialien zum 〈Nähren des Fötus〉 in Wilms 2005b; zu Scheinschwangerschaften, Wu 2002. Für einen Überblick des Themas von Fruchtbarkeit und Empfängnis, vgl. Riegel A-M 1999 (siehe die ausführliche Besprechung: Pfister, Rudolf, Asiatische StudienEtudes Asiatiques 60.4 (2006) 1072-1088. 
die 1117 von Yan Jizhong ediert wurde, gilt Qian Yi (auch Qian Zhongyang, 1032-1113, Song-Dynastie) als der Begründer der chinesischen Pädiatrie. Zumindest zeigt sich darin eine zunehmende Spezialisierung der GelehrtenÄrzte $(r u ́ y \bar{l}$ ), auch auf die Behandlung von Kindern. Laut der kurzen Studie von Flohr 2001 nimmt Qian ältere Ansichten über die Kinderheilkunde auf und systematisiert sie im ersten Teil; die so entwickelte pädiatrische Diagnostik und Pathologie wird dann anhand von dreiundzwanzig Fallgeschichten ${ }^{66}$ erläutert, und schliesslich führt Qian einhundertundvierzehn Rezepturen für Dekokte, Pillen, Salben, Pulver, Kekse und Säfte gegen ein breites Spektrum von Kinderkrankheiten wie Hautausschlägen, Durchfall, febrilen Erkrankungen usf. auf, insbesondere aber gegen 〈Krampfen〉 (fā chù), 〈Konvulsionen〉 (xián) sowie plötzliche oder langsam verursachte 〈Schreckanfälle〉 (jīng). Die kindliche Entwicklung im ersten Lebensjahr wird als stetige Abfolge aufgefasst, die in Schritten von zweiunddreissig Tagen abläuft. Solch ein zehnmaliges 〈Abändern〉 (biàn) und 〈Durchdämpfen〉 (zhēng) bewirke, von unten nach oben im Körper steigend, warmes Fliessgeschehen und dadurch auch organische und geistig-emotionale Veränderungen (wie die Hervorbringung der Willensinstanz, Präferenzen wie Freude und Wut, Intelligenz, erste Worte).

\section{Indische Einflüsse in der Ophthalmologie}

Allgemein sind Einflüsse von ausserhalb der Sinosphäre in die chinesische Heilkunde noch wenig behandelt worden, doch eine Reihe von kurzen Studien belegt die indischen Einflüsse auf die chinesische Augenheilkunde ${ }^{67}$ : Vijaya Deshpande zeigt am Fallbeispiel des Glaukoms oder grünen Stars (lü máng, qīng máng) die Aufnahme indischer Elemente in chinesische Medizintexte auf und beschreibt die ophthalmologische Chirurgie. Zu letzterer vergleicht er indische und chinesische Textstellen über die Behandlung von Pterygium (Flügelfell), Entropion oder Trichiasis (Einwärtskehrung der Lidränder bzw. der Wimpern) sowie von Katarakten. Zunächst in buddhistischen kanonischen Texten und später in spezialisierten ophthalmologischen Schriften übermittelt, die Nāgārjuna zugeschrieben wurden, hielten indische Elemente Einzug in die chinesische Medizin und legten so die

66 Siehe die Übersetzung der Fallgeschichten in Flohr 2001, 70-84.

67 Für die Übersetzung des spätkaiserzeitlichen Textes Des Silber-Meeres verfeinerte Subtilitäten (yín hăi jīng wēi), vgl. Kovacs/Unschuld 1998; darin S. 6-52 ein Abriss der Geschichte der Augenheilkunde und S. 37-39, 43-50 über indische Einflüsse auf die Ophthalmologie. 
Grundlage für die Einrichtung der Augenheilkunde als separater Disziplin in China ${ }^{68}$.

\section{Körpergeschichte}

«How can perceptions of something as basic and intimate as the body differ so?», lautet die Grundfrage in Kuriyama Shigehisas Versuch, einen direkten Vergleich des griechischen und chinesischen kulturellen Blicks auf den Körper und insbesondere auf die Muskulatur zu wagen ${ }^{69}$. Die Betonung von Differenz ergibt sich aus dieser Fragehaltung wie von selbst, beantwortet aber nicht, wie und was denn hier warum zur Vergleichung herausgehoben wird. Die Fahrt durch die Zeiten gelingt durch eine brillante und rasante Kontrastierung etwa der Stiläusserungen des Sehens (bei der Frage von Muskularität und Identität) und des Berührens (beim Pulsnehmen). Vielfach beschworen wird in der Literatur die Behauptung des Autors, das Chinesische hätte kein Wort für «Muskel» zur Verfügung gehabt, doch auf die Einteilung in 〈Flechsen〉 (jīn), die Sehnen und Muskeln vereint, und 〈Fleisch〉 (ròu), das seit der Schrift Über die Adern (mài shü) aus Zhangjiashan als Anhängsel der Knochen verstanden wird, geht man ebensowenig ein, wie Kuriyama die Dehnungsübungen zu Heilzwecken (siehe oben) oder die Kampfkünste bespricht, die sich doch wohl vorzüglich geeignet hätten, mit den Skulpturen der griechischen Athleten und den sportlichen Wettkämpfen kontrastiert zu werden. Wenn Kuriyama Sehstile und Illustrationen vergleicht, leitet dies auch über zur vermehrten Diskussion von Illustrationen und Bildmaterial innerhalb der Medizingeschichte Chinas ${ }^{70}$. Wie ist die Präzision des chinesischen Blickes auf Zwischenräume (zwischen Muskeln und Fleischteilen wie auch in der Bauchhöhle) sowie auf die Topographie der Körperhülle mit ihren kleinen Dellen und Öffnungen mit der Genauigkeit der anatomischen Untersuchung am toten Körper im Mittelmeerraum zu vergleichen? Sagt Kuriyama, dass das Anatomisieren der Griechen «a sort

68 Deshpande 1999, 2000, 2003-2004. Fan 2005 bespricht (ohne Kenntnis von Deshpandes Studien) die Interpretation der aus Indien eingeführten Technik des Katarakt-Stechens und deren Integration in die chinesische Medizin vom sechsten zum zwölften Jahrhundert u.Z. Ihm zufolge gelang die Aneignung, weil sie entlang der einheimischen chinesischen heilkundlichen Konzepte rekonstruiert werden konnte.

69 Kuriyama 1999. Eine knappe, jedoch kritische Besprechung gibt Paul Unschuld im Bulletin of the History of Medicine 75 (2001) 299-301.

70 Siehe dazu den Symposiumsband Kuriyama 2001 und den Bericht über die Konferenz Chinese Medicine: A Visual History von Lora-Wainwright 2005. Für visuelle Repräsentationen des Körpers aus dem Umfeld der daoistischen Religionsgemeinschaften vgl. Despeux 1994, $2005 b$. 
of spiritual desire» repräsentiere, so stellt sich sofort die Frage, wie denn auf chinesischer Seite das Geistige, das Denkvermögen und das Bewusstsein konzipiert worden seien ${ }^{71}$. All dies bedeutet, in noch kaum erkundete Bereiche hineinzufragen, und deutet damit das Mass der Anregung an, die sein Buch zu geben vermag.

\section{Bibliographie}

\section{Bibliographien, Übersichtsartikel}

Furth, Charlotte, "Bibliography of secondary sources on medicine and gender: early imperial China", Nan Nü 7.2 (2005) 309-316; Nachdruck in: Leung (2006) 201-208

Hinrichs, T. J., "New geographies of Chinese medicine", Osiris 14 (1998) 287-325

Lee, Jen-der, The Past as a Foreign Country: Recent Research on Chinese Medical History in Taiwan (Taipei 2003, http://www.ihp.sinica.edu.tw/ medicine/ashm/lectures/Jender\%20 Lee-ft.pdf)

Lessell, Colin B., Bibliotheca medica de Asia Orientali 1473-1900: an annotated chronological bibliography of western writings on Chinese and Japanese medicine, medicine of the adjacent territories and related topics (Bedford 2006)

Li, Jianmin/Kuriyama, Shigehisa/Zheng, Yaru (eds), shēntı̆shı̆ wénxiàn shūmù [Bibliographie der körpergeschichtlichen Literatur (Taipei 2000, http://www.ihp.sinica.edu.tw/ medicine/ book/book.htm)

Sivin, Nathan, "Science and medicine in imperial China - the state of the field", The Journal of Asian Studies 47.1 (1988) 41-90

\section{Monographien}

Cook, Constance A., Death in Ancient China: the Tale of One Man's Journey (Leiden 2006)

Despeux, Catherine, Taö̈sme et corps humain - le xiuzhen tu (Paris 1994)

- /Obringer Frédéric (direction), La maladie dans la Chine médiévale. La toux (Paris 1997)

Flohr, Carsten, Qian Yi: der Begründer der chinesischen Kinderheilkunde? (Würzburg 2001)

Furth, Charlotte, A Flourishing Yin. Gender in China's medical history, 960-1665 (Berkeley/Los Angeles, London 1999)

Goldin, Paul, The Culture of Sex in Ancient China (Honolulu 2002)

Goldschmidt, Asaf, The Transformations of Chinese Medicine during the Northern Song Dynasty (AD 960-1127) (Ph.D. Diss., University of Pennsylvania 1999)

Gulik, Robert Hans van [1910-1967], Erotic Colour Prints of the Ming Period, with an Essay on Chinese Sex Life from the Han to the Ch'ing Dynasty, B.C.206-A.D. 1644. Authorized reprint. With introductions by James Cahill, Wilt L. Idema and Sören Edgren (Leiden 2004) [Nachdruck der Ausgabe von 1951]

- Sexual Life in Ancient China. A preliminary survey of Chinese sex and society from ca 1500 B.C. till A.D. 1644. With a new introduction and bibliography by Paul R. Goldin (Leiden 2003) [Nachdruck der Ausgabe von 1961]

Harper, Donald J., Early Chinese Medical Literature: the Mawangdui Medical Manuscripts (London/New York 1998)

Hertzer, Dominique, Leuchten des Geistes und Erkenntnis der Seele: Die medizinische Vorstellung vom Seelischen als Ausdruck philosophischen Denkens - China und das Abendland (Frankfurt am Main 2006)

Hinrichs, T. J., The Medical Transforming of Governance and Southern Customs in Song China (960-1279 C.E.) (Ph.D. Diss., Harvard University 2003)

71 Einen globalen Vergleich der heilkundlichen Vorstellungen des Seelenlebens in China und dem Abendland macht Hertzer 2006. Siehe dazu auch die kurze Studie von Despeux 2007. 
Hinsch, Bret, Passions of the Cut Sleeve. The male homosexual tradition of China (Berkeley/Los Angeles/Oxford 1990)

Hsu, Elisabeth, The Telling Touch: Pulse diagnostics in early Chinese medicine. With translation and interpretation of 10 medical case histories of Shi ji 105.2 (ca 90 BC) (Habilitationsschrift im Fachbereich Sinologie, Fakultät für Orientalistik und Altertumswissenschaft, Universität Heidelberg 2001a)

- (ed.), Innovation in Chinese Medicine (Cambridge 2001b)

Kalinowski, Marc (éd.), Divination et société dans la Chine médiévale: Etudes des manuscrits de Dunhuang de la Bibliothèque Nationale de France et de la British Library (Paris 2003)

Keegan, David Joseph, The "Huang-ti nei-ching": the structure of the compilation; the significance of the structure (Ph.D. Diss., University of California, Berkeley 1988)

Kovacs Jürgen/Paul Ulrich Unschuld (translation and annotation), Essential Subtleties on the Silver Sea. The Yin-hai jing-wei: A Chinese Classic on Ophthalmology (Berkeley 1998)

Kuriyama, Shigehisa, The Expressiveness of the Body and the Divergence of Greek and Chinese Medicine (New York 1999)

- (ed.), The Imagination of the Body and the History of Bodily Experience (International Symposium 15; January 18-22, 2000) (Kyoto 2001)

Leung, Angela Ki Che (ed.), Medicine for Women in Imperial China (Leiden/Boston 2006) [Textidentischer Nachdruck der Zeitschrift Nan Nü, Men, Women, and Gender in China, Volume 7, Issue 2, 2005]

Lo, Vivienne, The Influence of Yangsheng Culture on Early Chinese Medical Theory (unpublished Ph.D. Thesis, University of London, Department of History, School of Oriental and African Studies 1998)

- /Cullen, Christopher (eds), Medieval Chinese Medicine. The Dunhuang medical manuscripts (London/New York 2005)

Middendorf, Ulrike, Resexualizing the Desexualized: the Language of Desire and Erotic Love in the Classic of Odes (Pisa/Rome 2007)

Needham Joseph/Lu Gwei-djen, Science and Civilisation in China, Volume 6 Biology and Biological Technology, Part VI: Medicine (Cambridge 2000)

Obringer, Frédéric, L'aconit et l'orpiment: drogues et poisons en Chine ancienne et médiévale (Paris 1997)

Pfister, Rudolf, der beste weg unter dem himmel. sexuelle körpertechniken aus dem alten china. zwei bambustexte aus mawangdui (Zürich 2003)

- sexuelle körpertechniken im alten China: seimbedürftige männer im umgang mit lebensspenderinnen: drei manuskripte aus Mawangdui: eine lektüre (Norderstedt, erscheint 2008)

Riegel, Andrea-Mercedes, Das Streben nach dem Sohn. Fruchtbarkeit und Empfängnis in den medizinischen Texten Chinas von der Hanzeit bis zur Mingzeit (München 1999) [zugleich: Dissertation, Ludwig-Maximilians-Universität München 1999]

Santangelo, Paolo/Guida, Donatella (eds), Love, Hatred and Other Passions: Questions and Themes on Emotion in Chinese Civilization (Leiden 2006)

Sartorius, Kurt (Hrsg.), Kolloquiumsbericht «Damit's Kind g'sund bleib» - Tabu Nachgeburtsbestattung - Kolloquium am 12. und 13. September 1997 in Bönningheim (Bönnigheim 2004)

Stein, Stephan, Zwischen Heil und Heilung: zur frühen Tradition des Yangsheng in China (Uelzen 1999)

Strickmann, Michel, Chinese magical medicine. Edited by Bernard Faure (Stanford 2002)

Umekawa, Sumiyo, Sex and Immortality - A Study of Chinese Sexual Activities for Better-Being (unveröffentlichte Dissertation, University of London, Department of History, School of Oriental and African Studies 2004)

Unschuld, Paul Ulrich, Huichun - Rückkehr in den Frühling. Chinesische Heilkunde in historischen Objekten und Bildern (München 1995)

- Huang Di nei jing su wen, Nature, Knowledge, Imagery in an Ancient Chinese Medical Text. With an Appendix The Doctrine of the Five Periods and Six Qi in the Huang Di nei jing su wen (with assistance by Zheng Jinsheng and Hermann Tessenow) (Berkeley/Los Angeles/ London 2003)

Vogel, Hans Ulrich/Moll-Murata, Christine/Gao, Xuan (eds), Studies on Ancient Chinese Scientific and Technical Texts. Proceedings of the 3rd ISACBRST. March 31-April 3, 2003 Tübingen, Germany [chinesisch/englisch] (Zhengzhou 2006) 
Wile, Douglas, The Chinese Sexual Yoga Classics Including Women's Solo Meditation Texts (Albany 1992)

Wilms, Sabine, The Female Body in Medieval Chinese Medicine: a Translation and Interpretation of the "Women's Recipes" in Sun Simiao's Beiji qianjin yaofang (Ph.D. Dissertation, University of Arizona 2002)

Yamada, Keiji, The Origins of Acupuncture, Moxibustion and Decoction. The two phases of the formation of ancient medicine: the origins of acupuncture and moxibustion. The origins of decoction (Kyoto 1998)

\section{Aufsätze}

Chen, Hsiu-fen, "Wind malady as madness in medieval China: some threads from the Dunhuang medical manuscript", in: Lo/Cullen (2005) 345-362

Collins, Roy/David Kerr,"The etymology of the word macrobiotic:s [sic] and its use in modern Chinese scholarship", Sino-Platonic Papers 112 (2001) 1-18

Deshpande, Vijaya, "Indian influences on early Chinese ophthalmology: glaucoma as a case study", Bulletin of the School of Oriental and African Studies 62 (1999) 306-322

- "Ophthalmic surgery: a chapter in the history of Sino-Indian medical contacts", Bulletin of the School of Oriental and African Studies 63.3 (2000) 70-388

- "Nāgārjuna et la Médecine Chinoise", Studia Asiatica. International Journal for Asian Studies IV-V (2003-2004) 241-258

Despeux, Catherine, "The system of five circulatory phases and the six seasonal influences (wuyun liuqi), a source of innovation in medicine under the Song (960-1279)", in:Hsu (2001b) 121-165

- «Auguromancie», in: Kalinowski (2003a) 431-470

- «Physiognomie», in: Kalinowski (2003b) 513-555

- «La gymnastique daoyin dans la Chine ancienne», Etudes chinoises 23 (2004) 45-86

- "From prognosis to diagnosis of illness in Tang China: comparison of the Dunhuang manuscript P. 3390 and medical sources", in: Lo/Cullen (2005a) 176-205

- "Visual representations of the body in Chinese medical and Daoist texts from the Song to the Qing period (tenth to nineteenth century)", translated by Penelope Barrett, Asian Medicine 1.1 (2005b) 10-52

- «Ames et animation du corps. La notion de shen dans la médecine chinoise antique», Extrême-Orient Extrême-Occident 29 (2007) 71-94

Drège, Jean-Pierre/Drettas, Dimitri, «Oniromancie», in: Kalinowski (2003) 367-404

Engelhardt, Ute, «Neue archäologische Funde zur Leitbahntheorie», Chinesische Medizin 13.3 (1998a) 93-100

- «Frühe archäologische Funde zum Qigong», in: Hildenbrand, Gisela/Manfred Geissler/ Stephan Stein (Hrsg.), Das Qi kultivieren, Die Lebenskraft nähren, West-Östliche Perspektiven zu Theorie und Praxis des Qigong und Yangsheng (Uelzen 1998b) 11-17

- «Daoyin tu und Yinshu: Neue Erkenntnisse über die Übungen zur Lebenspflege in der frühen Han-Zeit», Monumenta Serica 49 (2001a) 213-226

- "Dietetics in Tang China and the first extant works of materia dietetica", in: Hsu (2001b) 173-191

Fan, Ka Wai, "Couching for cataract and Sino-Indian medical exchange from the sixth to the twelfth century AD", Clinical and Experimental Ophthalmology 33.2 (2005) 188-190

Furth, Charlotte, "Rethinking Van Gulik: sexuality and reproduction in traditional Chinese medicine", in: Gilmartin, Christina K./Hershatter, Gail/Rofel, Lisa/White, Tyrene (eds), Engendering China: Women, Culture and the State (Cambridge 1994) 125-146

- "Rethinking van Gulik Again", Nan Nü 7.1 (2005) 71-78

Goldin, Paul, "Imagery of copulation in early Chinese poetry", Chinese Literature: Essays, Articles, Reviews 21 (1999) 35-66

- "The motif of the woman in the doorway and related imagery in traditional Chinese funerary art", Journal of the American Oriental Society 121.4 (2001) 539-548

- "The cultural and religious background of sexual vampirism in ancient China", Theology and Sexuality 12.3 (2006) 285-307 
Goldschmidt, Asaf, "Changing standards: tracing changes in acu-moxa therapy during the transition from the Tang to the Song dynasties", East Asian Science, Technology, and Medicine 18 (2001) 75-111

- "The song discontinuity: rapid innovation in northern song dynasty medicine", Asian Medicine: Tradition and Modernity 1 (2005) 52-90

Harper, Donald, "The sexual arts of ancient China as described in a manuscript of the second century B.C.", Harvard Journal of Asiatic Studies 47 (1987) 539-593

- "Iatromancy, prognosis, and diagnosis in early Chinese medicine", in: Hsu (2001) 99-120

- «Iatromancie», in: Kalinowski (2003) 471-512

- "Ancient and medieval Chinese recipes for aphrodisiacs and philters", Asian Medicine Tradition and Modernity 1.1 (2005a) 91-100

- "Dunhuang iatromantic manuscripts: P. 2856 R $^{\circ}$ and P. $2675 \mathrm{~V}^{\circ}$ ", in: Lo/Cullen (2005b) 134-164

He, Zhiguo/Lo, Vivienne, "The channels: a preliminary examination of a lacquered figurine from the Western Han Period", Early China 21 (1996) 81-123

Hinsch, Bret, "Van Gulik's sexual life in ancient China and the matter of homosexuality", Nan Nü 7.1 (2005) 79-91

Hsu, Elisabeth, "Pulse diagnostics in the Western Han: how mai and qi determine bing", in: Hsu (2001c) 51-91

- "Tactility and the body in early Chinese medicine", Science in Context 18.1 (2005) 7-34

Kalinowski, Marc, «Hémérologie», in: Kalinowski (2003a) 213-299

- «Cléromancie», in: Kalinowski (2003b) 301-368

- "Mantic texts in their cultural context", in: Lo/Cullen (2005) 109-133

Lee, Jen-der, "Wet nurses in early imperial China”, Nan Nü 2.1 (2000) 1-39

- "Gender and medicine in Tang China", Asia Major 16.2 (2003) 1-32

- "Childbirth in early imperial China", Nan Nü 7.2 (2005) 216-286, Nachdruck in: Leung (2006) 108-178

Li, Ling/McMahon, Keith, "The contents and terminology of the Mawangdui texts on the arts of the bedchamber", Early China 17 (1992) 145-185

Liu, Lexian, "Love charms among the Dunhuang manuscripts", translated by Vivienne Lo, in: Lo/Cullen (2005) 165-175

Lo, Vivienne, "Tracking the pain. Jue and the formation of a theory of circulating $q i$ through the channels", Sudhoffs Archiv 83 (1999) 191-211

- "The influence of Western Han nurturing life literature on the development of Acumoxa therapy", in: Hsu (2001) 19-51

- "Spirit of stone: technical considerations in the treatment of the jade body", Bulletin of the School of Oriental and African Studies 65.1 (2002) 99-128

- "Quick and easy Chinese medicine: the Dunhuang moxibustion charts", in: Lo/Cullen (2005) 211-222 figures, 227-252

Lora-Wainwright, Anna, "Chinese Medicine: a Visual History. September 14th-16th 2005, Fragrant Hills Hotel, Beijing, China”, IASTAM Newsletter, December (2005) 28-33

Mayanagi, Makoto, "The three juan edition of Bencao jizhu and excavated sources", translated by Sumiyo Umekawa, in: Lo/Cullen (2005) 306-321

Mollier, Christine, "Talismans", in: Kalinowski (2003) 405-429

Obringer, Frédéric, "A song innovation in pharmacotherapy: some remarks on the use of white arsenic and flowers of arsenic", in: Hsu (2001) 192-213

Pfister, Rudolf, «Sexuelle Körpertechniken in den medizinischen Manuskripten aus Mawangdui», Asiatische Studien - Etudes Asiatiques 51.4 (1997) 1039-1046

- "Some preliminary remarks on notational systems in two medical manuscripts from Mawangdui”, Asiatische Studien - Etudes Asiatiques 56.3 (2002) 609-633

- "The production of special mental states within the framework of sexual body techniques as seen in the Mawangdui Medical Corpus", in: Santangelo/Guida (2006a) 180-194

- "The jade spring as a source of pleasure and pain: the prostatic experience in ancient and medieval medical and Daoist texts", in: Vogel/Moll-Murata/Gao (2006b) 88-106

- «Der Milch-Baum und die Physiologie der weiblichen Ejakulation: Bemerkungen über Papiermaulbeer- und Feigenbäume im Süden Altchinas», Asiatische Studien - Etudes Asiatiques 61.3 (2007) 813-844 
Riegel, Andrea-Mercedes, «Das Verbergen der Nachgeburt (zang [recte cáng] bao) - ein Thema der Medizin im antiken China», in: Sartorius Kurt (2004) 84-87

Riegel, Jeffrey K., "Eros, introversion, and the beginning of the Shijing commentary", Harvard Journal of Asiatic Studies 57.1 (1997) 143-178

Sakade, Yoshinobu, "Daoism and the Dunhuang regimen texts", translated by Sumiyo Umekawa, in: Lo/Cullen (2005) 278-290

Tessenow, Hermann, "The Huang Di Neijing Suwen project - methodology of style analysis", Asiatische Studien - Etudes Asiatiques LVI.3 (2002) 647-658

Umekawa, Sumiyo, "Tiandi yinyang jiaohuang dalefu and the art of the bedchamber", in: Lo/Cullen (2005) 252-277

Unschuld, Paul Ulrich/Zheng, Jinsheng, «Handschriften als Quellen chinesischer Medizingeschichte», Monumenta Serica 48 (2000) 471-494

- "Manuscripts as sources in the history of Chinese medicine", translated from German by Mitch Cohen, in: Lo/Cullen (2005) 19-44

Wang, Shumin, "A general survey of medical works contained in the Dunhuang medical manuscripts", translated by Vivienne Lo, in: Lo/Cullen (2005a) 45-58

- "Appendix II: Abstracts of the medical manuscripts from Dunhuang", translated by Penelope Barrett, in: Lo/Cullen (2005b) 374-434

- "The Dunhuang manuscripts and pharmacology in medieval China", translated by Christopher Cullen, in: Lo/Cullen (2005c) 293-305

- "Tangye jingfa (canonical methods for brews and decoctions): a lost text recorded in the Hanshu bibliography", translated by Chen Hsiu-fen, in: Lo/Cullen (2005d) 322-344

Wilms, Sabine, "The art and science of menstrual balancing in early medieval China", in: Shail, Andrew/Howie, Gillian (eds), Menstruation: a Cultural History (Basingstoke, Hampshire/ New York 2005a) 38-50

- "The transmission of medical knowledge on "nurturing the fetus' in early China", Asian Medicine. Tradition and Modernity 1.2 (2005b) 277-314

- "Ten times more difficult to treat, female bodies in medical texts from early imperial China", Nan Nü 7.2 (2005c) 181-215, Nachdruck in: Leung (2006a) 74-107

- "The formation and textual knowledge in the development of medieval gynecology. The sources for the second through fourth scrolls of Sun Simiao's Beiji qianjin yaofang" in: Vogel/Moll-Murata/Gao (2006b) 107-134

$\mathrm{Wu}$, Yi-li, "Ghost fetuses, false pregnancies, and the parameters of medical uncertainty in classical Chinese gynecology", Nan Nü 4.2 (2002) 170-206

Xie, Guihua, "Han bamboo and wooden medical records discovered in military sites from the northwestern frontier regions", translated by Vivienne Lo, in: Lo/Cullen (2005) 78-106

Yamada, Keiji, "The formation of the 'Huang-ti nei-ching"”, Acta Asiatica 36 (1979) 67-89

Yates, Robin D. S., "Medicine for women in early China: a preliminary survey”, Nan Nü 7.2 (2005) 127-181, Nachdruck in: Leung (2006) 19-73

Zheng, Yan'e, "Măwángduī Hànmù wénxiàn yàomù" [Verzeichnis der wesentlichen Schriften zu den Gräbern von Mawangdui], in: He, Jiejun, et al., Chángshā Măwángduì èr, sān hào Hànmù, dì ȳ juàn: tiányĕ kăogŭ fājué bàogào - Tombs 2 and 3 of the Han Dynasty at Mawangdui, Changsha Report on Excavation, volume 1 (with abstracts in English and Japanese) (Beijing 2004) 281-381 